\title{
Models for apparent reaction kinetics in heap leaching: a new semi-empirical approach and its comparison to shrinking core and other particle-scale models
}

\author{
Richard J. Ferrier, Liping Cai, Qingyang Lin, Gerard J. Gorman, Stephen J. Neethling ${ }^{1}$ \\ Rio Tinto Centre for Advanced Mineral Recovery, Department of Earth Science and Engineering, Imperial \\ College London, SW7 2AZ, UK \\ ${ }^{1}$ Corresponding author. E-mail: s.neethling@imperial.ac.uk
}

October 2015

\begin{abstract}
Particle-scale effects are critically important to the performance of heap leaching operations. In a heap-scale simulation, the transport of fluid phases and reactive species external to the ore particles might be modelled with thousands of grid elements by the finite volume or finite element method. The inter- and intra-particle diffusions and reactions are usually parametrised by a deterministic model, such as the shrinking core model (SCM), that translates the external conditions into an effective product extraction rate. However, the rate equation takes the form of an implicit or partial differential equation for all but the simplest models and kinetic regimes, becoming expensive to solve on large grids. We instead propose an economical, easily calibrated semi-empirical approach in which the dependencies on external conditions and the current state of the ore are considered to be mathematically separable. We show that the standard SCM does not suffer greatly from this approximation even when there is a mixed control regime with nonlinear kinetics. The dependency on the state of the ore is derived empirically, inherently capturing heterogeneous features and cluster-scale effects. We demonstrate that this method scales correctly when fitted to data from physical column leaching experiments.
\end{abstract}

\section{Introduction}

Heap leaching is simple means of extracting valuable metals from low grade ore. With relatively low capital and operational costs, it has become increasingly popular in the mining industry. However, the design of heap leaching operations is far from optimal. Predictive models are needed, of which Bartlett (1992) defines two types: empirical and deterministic. Empirical models rely on historical data to parametrise a particular heap. These are valid provided there is enough data covering a large enough parameter space. Unfortunately, factors such as the properties of the ore mean that successful empirical models are likely to be site-specific at best. With so many variables involved in heap leaching operations, and comparatively little historical data available, scaling up the models to larger heaps is unfeasible and the cost on a per-installation basis remains high (Dixon, 2000).

In the absence of effective industrial empirical models, the research community has proposed various deterministic models for heap leaching systems. Deterministic models are created from first principles using what are believed to be the dominant physicochemical factors of the problem. However, the complete heap leaching system is complex and multiscale, with many interacting mechanisms at work. This makes the modelling of the system a challenging proposition. Some of the processes that are important include the transport of fluid through the packed bed, the mass transport within the ore particles and reactions such as the dissolution of metals and other species taking place at the mineral grain surfaces. The prediction of the behaviour of these systems is often complicated by the further interactions with processes such as biological activity, gas motion and heat transport. Heap leaching models that capture these processes have been developed by, for instance, Dixon (2000) and Leahy et al. (2006).

The transport processes and chemical reactions occurring within the ore particle are modelled together, with the mineral grains assumed to be dispersed evenly throughout the ore such that the entire solid may be considered to act as a homogeneous reactant. The emergent rate of conversion is dependent upon the current state of the leaching and the concentration of the reagents in the fluid surrounding the ore particle. This intra-particle modelling is usually done by means of the shrinking core model (SCM) or one of its variants. In the standard SCM (Yagi and Kunii, 1955), each particle is assumed to be a sphere of initially unreacted material. Reaction takes place on the surface of the sphere and then progresses inwards, such that the core of unreacted material shrinks and leaves behind a layer of inert, permeable, solid product. This situation is representative of leaching when there is a thin reaction zone (Braun et al., 1974; Roman et al., 1974). Under further assumptions an explicit ordinary differential equation (ODE) can be formed from which the product extraction rate may be calculated straightforwardly, otherwise an implicit and/or partial differential equation must be resolved. 
An alternative model is the homogeneous or 'progressive conversion' model (PCM) (Wen, 1968; Levenspiel, 1972), which is simply the solution of the diffusion-reaction equation in spherical coordinates. This model is appropriate when there is a broad reaction zone (Bartlett, 1973). The solid reagent distributed throughout the particle is now depleted gradually according to the local fluid reagent concentration. The model must be solved numerically with, for example, finite differences owing to the coupling between concentration profiles.

One problem with these models is the computational expense. If the governing equation of the particle scale model cannot be reduced to an explicit ODE, the overall conversion rate must be solved for implicitly. Typical heap scale simulations in $2 \mathrm{D}$ or $3 \mathrm{D}$ involve thousands of grid elements, and the conversion rate must be determined for each of these at every time step. This explains why many heap scale models presented in the literature are coupled to explicit SCM formulations (for example Leahy et al., 2005; Bennett et al., 2012).

Another problem is that deterministic models are only as good as their inputs and assumptions, and in the context of heap leaching these can be especially difficult to justify. In particular, it may be inappropriate to couple a heap scale model - describing multiphase fluid transport in two or three dimensions - directly to the SCM under the assumption that the fluid properties provide the particle boundary conditions. More recent studies suggest a dual porosity visualisation in which clusters of particles make up stagnant fluid zones connected by a sparse network of flow channels (Bouffard and Dixon, 2001; Petersen and Dixon, 2007; Ghorbani et al., 2011). At this scale, communication of the reactive species is by diffusion alone and the PCM is arguably a more appropriate choice of model. Heap leaching models that rely on the PCM include, for example, HeapSim (Ogbonna et al., 2005). Those that rely on the SCM must extend the scope of the model by changing the physical meaning of the parameters; for example Miller (2003) replaces the individual particle size with an effective particle size.

The standard SCM and PCM may be deficient even when applied to individually leached particles. Ghorbani et al. (2011) point out that unique to heap leaching is the relatively large particle size. These particles may have cracks and other heterogeneities, affecting the tortuosity; they may have an irregular makeup of mineral grains; and they may break up over the course of leaching. The SCM and PCM have only a few parameters, such as the effective diffusivity of reagents through the inert layer, to encapsulate these features. Ghorbani et al. question whether the SCM is necessarily valid under these circumstances.

Researchers have nevertheless succeeded in validating heap leaching models that are directly coupled to shrinking core models. A common approach is to calibrate the effective particle sizes, rate constants and other parameters empirically from small-scale laboratory experiments using a given ore sample (Cross et al., 2006; Bennett et al., 2012; McBride et al., 2012). Subsequent predictive simulations will be valid for that class of ore only, and often only for the chemical conditions used during the calibration. By extension, because ore properties are so sitespecific, the simulations will usually only be valid for the corresponding site. This approach makes sense from an industrial point of view: a one-time calibration per site is a small price to pay for better predictive capability. However, choosing the parameters to best fit the laboratory results will be an iterative process if the particles follow a size distribution rather than being uniformly sized.

In this paper, we propose an alternative model which is empirical in the sense that no assumption is made about the geometry, physical structure or mineral grain distribution of the particles taking part in the leach process. The key assumption is that the effect of the current state of the ore, characterised by, for instance, the extent of the reaction $X$, is mathematically separable from the conditions to which the ore particles are exposed (chemical concentrations, temperature, $\mathrm{pH}$, Eh, wetting etc.). A similar model for the intrinsic particle leach kinetics was proposed by Dixon and Hendrix (1993a) and extended by Ghorbani et al. (2013). That model takes the form of a product of functions which is substituted into the source term of the PCM. However, we make an even broader approximation by replacing the entire partial differential equation with a product of functions, hence cutting the computational cost.

In terms of the dependency on the current state of the ore, our method differs from the SCM and PCM in that, instead of using a set of parameters calculated a priori or calibrated a posterior $i$ to best fit a given extraction curve ( $X$ against time), we calibrate a function $f(X)$ such that the experimental extraction curve is matched by construction. This approach was first introduced by Cai et al. (2014). For the present work, we go further in validating the method and comparing it against existing models.

In the next section we explore several existing models, including the standard SCM and PCM. With the SCM, one is forced to solve an implicit equation when the reaction kinetics are nonlinear. We show that it is possible to replace this equation by an explicit equation without greatly affecting the resulting extraction curve. We use this argument to support our proposed semi-empirical approach and the assumption of separability. The final part of the paper is a case study showing how the various models may be calibrated against experimental data, and how they scale to data corresponding to different reagent concentrations.

The case study features the leaching of chalcopyrite. Chalcopyrite is the most abundant copper mineral and is attracting increasing interest as a leaching target. It also features prominently in the literature (Braun et al., 1974; Munoz et al., 1979). However, we emphasise that the proposed methodolody is general and can be applied just as easily to the leaching of other minerals. 


\section{Existing Models}

In this section, variations on the shrinking core model and progressive conversion model are described. These variants appear in the literature in the context of individual particle leaching, and are validated using flask or tank leaching experiments rather than with columns or heaps. As noted above, this does not preclude the models from being extended to the cluster scale in heap leaching simulations. However, the physical meanings of some of the model parameters may change.

A chemical reaction is considered to take place between liquid reagent $A$ and solid reactant $B$ according to the formula

$$
A+b B \rightarrow \text { products, }
$$

where $b$ is the stoichiometric number. Reagent $A$ penetrates the ore with an effective diffusivity of $D_{e}$. $B$ is assumed to be dispersed homogeneously throughout the ore particle or cluster so that the entire solid or stagnant zone can be modelled as a single reactant. Both the reagent and reactant concentrations, $C_{A}$ and $C_{B}$, have units of molar amount per unit volume of ore. The ore particles or clusters are considered to be spherical with a representative radius $r_{0}$ and porosity $\varepsilon_{0}$.

The effective diffusivity in a particle is lower than the diffusivity within a body of free water $D$, on account of the reduced fluid volume fraction and the constrictivity and tortuosity of pores connecting the reactant zones. This relationship is given by (Saripalli et al., 2002)

$$
D_{e}=\frac{D \delta \varepsilon_{0}}{\tau}
$$

where $\delta$ and $\tau$ are factors representing the constrictivity and tortuosity, respectively. $D_{e}$ can be difficult to calculate owing to the high level of uncertainty associated with the factors in Equation (1). To make matters worse, in the context of heap leaching the intra-particle diffusion needs to be lumped together with diffusion in the stagnant zones between particles, such that $D_{e}$ now represents the effective diffusivity of the path travelled by reageants and leached product. In this case it may be better to calculate $D_{e}$ a posteriori from experiments, or use $D$ unmodified and instead estimate an effective particle size as Miller (2003) did.

For the present study, we do not attempt to derive $D_{e}$ or other physical parameters at all, preferring instead to reduce the parameters to a set of nondimensional numbers. These will be calibrated using least-squares minimisation so that the resulting product extraction curve optimally fits a reference set of experimental column leaching data.

In the spirit of Wen (1968) and Dixon and Hendrix (1993a), we nondimensionalise the concentrations, radius $r$ and time $t$ as

$$
C=\frac{C_{A}}{C_{A 0}}, \quad \quad \quad \quad \xi=\frac{C_{B}}{C_{B 0}}, \quad t^{*}=\frac{t}{r_{0}}, \quad
$$

where $t_{D}=\varepsilon_{0} r_{0}^{2} / D_{e}$, representing a characteristic diffusion time, and $C_{A_{0}}$ and $C_{B 0}$ are reference concentrations. We further introduce a dimensionless external reagent concentration $C_{\text {ext }}$, stoichiometric ratio $\beta$, shrinking core reaction modulus $\kappa_{c}$ and interior pore reaction modulus $\kappa_{p}$ :

$$
\begin{aligned}
C_{\mathrm{ext}}=\frac{C_{A \mathrm{ext}}}{C_{A 0}}, \quad \kappa_{c}=\frac{k_{s} r_{0} C_{A 0}{ }^{n-1} C_{B 0}{ }^{m}}{b D_{e}}, \\
\beta=\frac{\varepsilon_{0} b C_{A 0}}{C_{B 0}}, \quad \kappa_{p}=\frac{k_{p} r_{0}^{2} C_{A 0}{ }^{n-1} C_{B 0}{ }^{m}}{b D_{e}},
\end{aligned}
$$

where $C_{A \text { ext }}$ is the concentration of $A$ surrounding the particles, $k_{s}$ is a reaction rate constant expressed per unit particle surface area, $k_{p}$ is expressed per unit particle volume and $n$ and $m$ are the orders of reaction. The reaction moduli $\kappa_{c}$ and $\kappa_{p}$ represent the ratio of reaction rate to diffusion rate and are equivalent to Damköhler II numbers.

\subsection{Shrinking core models}

In the shrinking core model, the reaction zone is considered to be thin, vanishing at some dimensionless core radius $\xi_{c}$. Prognosis of $S$ becomes trivial: $S=1$ where $\xi<\xi_{c}$ and $S=0$ otherwise. This means that extraction $X$, or the extent of conversion by volume, can be calculated from $\xi_{c}$ by

$$
X=1-\xi_{c}^{3}
$$

The standard SCM (Yagi and Kunii, 1955) is able to account for three mechanisms which determine the overall rate of conversion of the solid reactant. These mechanisms are: (i) mass transfer from the ambient fluid through the fluid film surrounding the particle; (ii) diffusion through the inert product layer left behind as the core shrinks; and (iii) chemical reaction at the surface of the unreacted core. Because mechanism (i) tends to have relatively little impact in heap leaching, it will be ignored in this paper. 
The governing equation for the shrinking core model may now be written

$$
\frac{\partial C}{\partial t^{*}}=\frac{1}{\xi^{2}} \frac{\partial}{\partial \xi}\left(\xi^{2} \frac{\partial C}{\partial \xi}\right)
$$

which is subject to the boundary conditions

$$
\begin{aligned}
C & =C_{\text {ext }} & \text { at } \xi & =1, \\
\frac{\partial C}{\partial \xi} & =\kappa_{c} C^{n} & \text { at } \xi & =\xi_{c} \\
\frac{\partial C}{\partial \xi} & =-\frac{1}{\beta} \frac{\mathrm{d} \xi_{c}}{\mathrm{~d} t^{*}} & \text { at } \xi & =\xi_{c}
\end{aligned}
$$

and initial condition

$$
\xi_{c}=1 \quad \text { at } t^{*}=0
$$

where $\xi_{c}$ is the dimensionless core radius.

The modelling is simplified if $\partial C / \partial t^{*}$ is neglected. This is the "pseudo-steady state" assumption, and is generally valid as long as $\beta$ is small (Wen, 1968; Liddell, 2005). The partial differential equation then reduces to an implicit ordinary differential equation,

$$
\frac{1}{\beta} \frac{\mathrm{d} \xi_{c}}{\mathrm{~d} t^{*}}+\kappa_{c}\left[C_{\mathrm{ext}}+\xi_{c}\left(1-\xi_{c}\right) \frac{1}{\beta} \frac{\mathrm{d} \xi_{c}}{\mathrm{~d} t^{*}}\right]^{n}=0
$$

Equation (7) may be integrated numerically using an implicit differential algebraic solver. Alternatively, if the overall rate of conversion is considered to be reaction-controlled $\left(\kappa_{c} \approx 0\right)$ or diffusion-controlled $\left(\kappa_{c} \gg 1\right)$, or if the reaction kinetics are linear $(n=1)$, the equation can be made explicit and integrated analytically to give $t^{*}\left(\xi_{c}\right)$.

Some researchers have modified the standard SCM to capture phenomena that are peculiar to the leaching process. Braun et al. (1974) observed an enhanced leaching rate in the later stages of one of their experiments. They attributed this to the generation of cracks and fissures which can occur especially in large particles. The enhanced leaching rate can be represented in the SCM via a particle shape factor $\phi$ that effectively scales the particle radius and hence $\mathrm{d} \xi_{c} / \mathrm{d} t^{*}$. Originally the shape factor was introduced to correct for particle surface roughness and deviation of the particle shape from sphericity. However, Braun et al. define its change with radius as proportional to the interfacial area,

$$
\frac{\mathrm{d} \phi}{\mathrm{d} \xi} \propto \frac{\xi^{2}}{\phi}
$$

thereby providing a means of slightly accelerating the extraction as the simulation progresses. A constant of proportionality needs to be determined.

The model can also be modified to correct for preexisting heterogeneities in the solid. Velardo et al. (2002) argue that the assumption of homogeneous particles is one of the main weaknesses of the SCM in the context of leaching. In reality, mineral grains tend to be more accessible towards the particle surface where the wetted surface-to-volume ratio of the porous solid is larger. To account for this, Velardo et al. propose a two-layer model where $\kappa_{c}$ is modified. In the inner layer $\left(\xi<\xi_{i}\right), \kappa_{c}$ keeps its original value. In the outer layer $\left(\xi_{i}<\xi_{c}<1\right)$, it changes according to some smooth step function $h(\xi)$ to a maximum of $\alpha_{1}$ times its original value. Thus

$$
\kappa_{c}^{\prime}=\left[1+\left(\alpha_{1}-1\right) h(\xi)\right] \kappa_{c}
$$

where

$$
h(\xi)= \begin{cases}0 & \xi<\xi_{i} \\ \exp \left[\alpha_{2}\left(1+\frac{\left(1-\xi_{i}\right)^{2}}{(1-\xi)^{2}-\left(1-\xi_{i}\right)^{2}}\right)\right] & \xi_{i}<\xi<1\end{cases}
$$

Therefore, three new dimensionless parameters are introduced: a scale factor $\alpha_{1}$, a steepness factor $\alpha_{2}$ and the inner layer radius $\xi_{i}$.

Most of the studies validating the SCM in the context of leaching have been supported by the tank leaching of finely milled particles (Braun et al., 1974; Neuburg et al., 1991). We reiterate that the SCM has been successfully applied in heap leaching simulations to capture effects occurring at both particle and cluster scales. Leahy et al. (2005) use the diffusion- and surface reaction-controlled SCM which is then coupled to equations involving bacteria-catalysed reactions. Bennett et al. (2012) also use this SCM but replace the standard surface reaction term by an empirical, nonlinear function of $C_{\text {ext }}, X$ and temperature. 
In the homogeneous or progressive conversion model, the whole particle is considered to be the reaction zone. A core radius is not usually modelled. $S$ now becomes a prognostic variable which is coupled to $C$ via a source term added to Equation (4).

For generality, the model of Dixon and Hendrix (1993a) is described here. Dixon and Hendrix point out that while most mineral grains can be found lining the pores of the ore particles, a proportion $\lambda$ may reside on the outer surface. Therefore, in addition to solving for a porous solid concentration $S_{p}$ inside the particle (or equivalently, inside the particle cluster), we also solve for a solid concentration $S_{s}$ at the surface.

The overall extraction $X$ may be integrated numerically according to

$$
X=3(1-\lambda) \int_{0}^{1}\left(1-S_{p}\right) \xi^{2} \mathrm{~d} \xi+\lambda\left(1-S_{s}\right)
$$

The governing equations are

$$
\begin{aligned}
\frac{\partial C}{\partial t^{*}} & =\frac{1}{\xi^{2}} \frac{\partial}{\partial \xi}\left(\xi^{2} \frac{\partial C}{\partial \xi}\right)-\kappa_{p} C^{n} S_{p}^{m_{p}} \\
\frac{\mathrm{d} S_{p}}{\mathrm{~d} t^{*}} & =-\frac{\beta \kappa_{p}}{1-\lambda} C^{n} S_{p}^{m_{p}} \\
\frac{\mathrm{d} S_{s}}{\mathrm{~d} t^{*}} & =-\frac{\beta \kappa_{s}}{\lambda} C_{\mathrm{ext}}^{n} S_{s}^{m_{s}}
\end{aligned}
$$

where, like $\kappa_{c}, \kappa_{s}$ is a rate constant expressed per unit particle surface area. If the mineral grains at the surface have the same reactivity as those in the interior, one may assume that $\kappa_{s}=\frac{\lambda}{1-\lambda} \kappa_{p}$ and $m_{s}=m_{p}$.

Equations (12) and (13) must be discretised in space as well as integrated in time. Central finite differences with second-order accuracy are a natural choice (Bartlett, 1973; Dixon and Hendrix, 1993a). The boundary conditions are

$$
\begin{aligned}
C & =C_{\text {ext }} & \text { at } \xi & =1, \\
\frac{\partial C}{\partial \xi} & =0 & \text { at } \xi & =0,
\end{aligned}
$$

and the initial conditions are

$$
\begin{array}{ll}
S_{p}=S_{s}=1 & \text { at } t^{*}=0, \\
C=0 & \text { at } t^{*}=0 .
\end{array}
$$

The above model reduces to the standard PCM when $\lambda=0$.

In another variant, Wen (1968) presents a hybrid with the SCM whereby a shrinking core radius $\xi_{c}$ is once again solved for. Reactions take place inside the core $\left(\xi<\xi_{c}\right)$, leaving behind a fully reacted outer layer. In limiting cases, depending on the effective diffusivity, the model reduces to the standard SCM or PCM.

Dixon and Hendrix (1993b) show that different mineral grain distributions may be represented by adjusting $m_{p}$ or $m_{s}$. The classical shrinking sphere model is recovered when $m=2 / 3$, and broader log-normal distributions can be approximated by choosing higher values.

Bouffard and Dixon (2001) consider a dimensional form of Equation (13), referring to this as the K- $\varphi$ model.

$$
\frac{\mathrm{d} X}{\mathrm{~d} t}=K(1-X)^{\varphi}
$$

Here $X$ is synonymous with $S_{p}$, and $\varphi$ is synonymous with $m_{p}$. Ghorbani et al. (2013) extend the model by introducing a new parameter $\alpha_{1}$ representing the readily leachable fraction of reactant. They then integrate Equation (17) and add a linear term of gradient $\alpha_{2}$ to describe the apparent linear extraction of the poorly leachable fraction in the later stages.

$$
X= \begin{cases}\alpha_{1}\left[1-\left(1+(\varphi-1) \frac{K t}{\alpha_{1}}\right)^{\left.\frac{1}{1-\varphi}\right]+\alpha_{2} t}\right. & t \leq \frac{\alpha_{1}}{K(1-\varphi)} \text { if } \varphi<1 ; \text { all } t \text { if } \varphi>1 \\ \alpha_{1}+\alpha_{2} t & t \leq \frac{\alpha_{1}}{K(1-\varphi)} \text { if } \varphi<1\end{cases}
$$




\section{Separation of the Shrinking Core Model Equation}

In $\S 2.1$, it was pointed out that the implicit equation of the pseudo-steady SCM could be reduced to an explicit equation if the system was considered either wholly reaction-controlled or wholly diffusion-controlled, or if the reaction kinetics were linear. An explicit equation has the advantage of being computationally economical when coupled to a heap scale model with many grid elements and time steps.

However, it is sometimes the case, especially in chalcopyrite leaching, that reaction and diffusion have comparable influence and that the reaction kinetics are nonlinear (Madsen and Wadsworth, 1980; Kimball et al., 2010). We propose that in such a situation Equation (7) may be approximated by a modification to the SCM with linear kinetics. We shall refer to this approximant as the separated SCM, since the intrinsic, nonlinear dependence on $C_{\text {ext }}$ is separated from the dependence on the extent of conversion characterised by $\xi_{c}$ or $X$.

With linear reaction kinetics $(n=1)$, Equation (7) reduces to

$$
\frac{1}{\beta} \frac{\mathrm{d} \xi_{c}}{\mathrm{~d} t^{*}}=\frac{C_{\mathrm{ext}}}{1 / \kappa_{c}+\xi_{c}\left(1-\xi_{c}\right)} .
$$

Suppose the linear scaling on $C_{\text {ext }}$ is now replaced with some nonlinear scaling, $C_{\text {ext }}^{n^{\prime}}$ :

$$
\frac{1}{\beta} \frac{\mathrm{d} \xi_{c}}{\mathrm{~d} t^{*}}=\frac{C_{\mathrm{ext}}^{n^{\prime}}}{1 / \kappa_{c}+\xi_{c}\left(1-\xi_{c}\right)},
$$

where $n^{\prime}$ must be chosen such that Equation (20) matches the behaviour of (7) as closely as possible. By inspection, $n^{\prime}$ will approach $n$ when reaction-controlled $\left(\kappa_{c} \approx 0\right)$ and unity when diffusion-controlled $\left(\kappa_{c} \gg 1\right)$.

Equation (20) can be integrated exactly as

$$
\begin{aligned}
t^{*} & =\frac{1}{\beta C_{\mathrm{ext}}^{n^{\prime}}}\left[\frac{1-\xi_{c}}{\kappa_{c}}+\frac{1-3 \xi_{c}^{2}+2 \xi_{c}^{3}}{6}\right] \\
& =\frac{1}{\beta C_{\mathrm{ext}}^{n^{\prime}}}\left[\frac{1-(1-X)^{1 / 3}}{\kappa_{c}}+\frac{1-3(1-X)^{2 / 3}+2(1-X)}{6}\right] .
\end{aligned}
$$

An informal analysis quantifying the maximum error in $X$ associated with Equation (21) is given below. This may be of interest to researchers who need to represent nonlinear kinetics in their heap leaching simulations and who wish to use an economical alternative to the implicit SCM, but are concerned about the loss in accuracy. The analysis also helps validate the assumption of separability underpinning the semi-empirical model presented in the next section.

\subsection{Calculation of the apparent order of reaction}

$n^{\prime}$ was computed numerically for a range of $n, C_{\text {ext }}$ and $\kappa_{c}$ as follows. For each combination of parameter values, Equation (7) was integrated over $\beta t^{*}$ using the Assimulo differential algebraic solver (Andersson et al., 2014). $n^{\prime}$ was then tuned so that Equation (21) gave the smallest least squares error in $X$ compared to the standard model's curve. The Brent algorithm implemented in SciPy (Jones et al., 2001) was used for this optimisation. Errors were sampled over the full extraction range $(0 \leq X \leq 1)$.

As an example, consider the case of $n=0.42$ and $C_{\text {ext }}=0.2$. This value of $n$ is representative of the copper leaching of chalcopyrite by ferric sulphate (Kimball et al., 2010), while the value of $C_{\text {ext }}$ represents the scaling between column experiments which feature in $\S 5$.

Figure 1 shows how $n^{\prime}$ and the associated extraction errors $\left|X^{\prime}-X\right|$ vary over the range of $\kappa_{c}$. As expected, $n^{\prime}$ approaches 0.42 and 1.0 for the limiting cases of $\kappa_{c} \approx 0$ and $\kappa_{c} \gg 1$, with the error between the two models approaching zero in both cases. The peak errors occur at $\kappa_{c} \approx 10$. Even in this worst case, it can be seen that the overall behaviour of the separated SCM is still close to that of the implicit model, with a maximum error of $5 \%$ and time-averaged error of $2 \%$. The $R^{2}$ coefficient of determination is favourable, at $99 \%$. The extraction curves for this intermediate case are shown in Figure 2.

The peak errors will generally increase as $n$ and $C_{\text {ext }}$ deviate from unity. Figure 3 shows the peak maximum error for a range of $C_{\text {ext }}$ and selected values of $n$. It can be seen that for modest orders of reaction $(0.5 \leq n \leq 2)$, scaling the external concentration up or down by a factor of 10 is accompanied by extraction errors of less than $10 \%$.

In a heap leaching simulation, the reference concentration $C_{A 0}$ would be calibrated based on the typical saturation within the heap, so $C_{\text {ext }}$ would not deviate far from unity except at fringe regions or for highly unsteady flow. Therefore, one might expect the simulated total extraction to suffer much less of an error than the values suggested in the above analysis. 


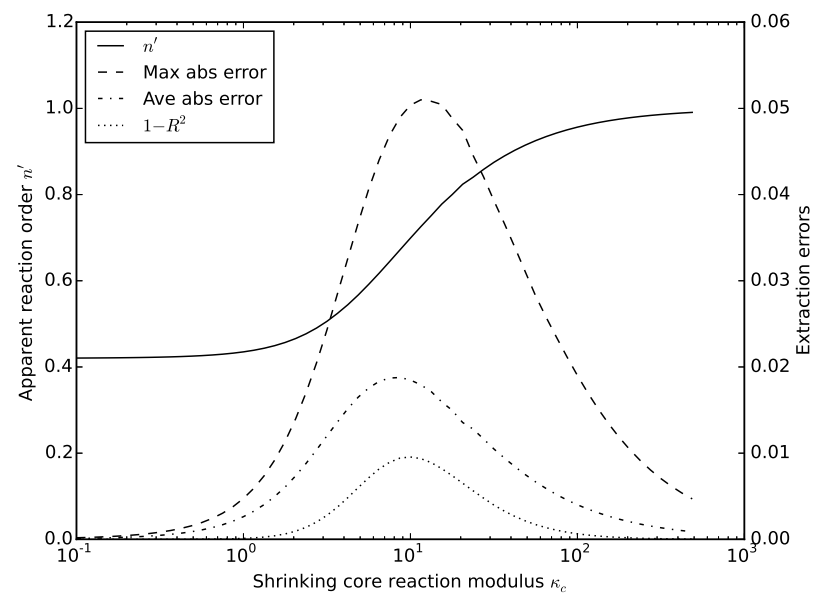

Figure 1: Optimisation of an apparent order of reaction for the pseudo-steady SCM over a range of reaction moduli (reaction-controlled through diffusion-controlled regimes), with associated extraction errors

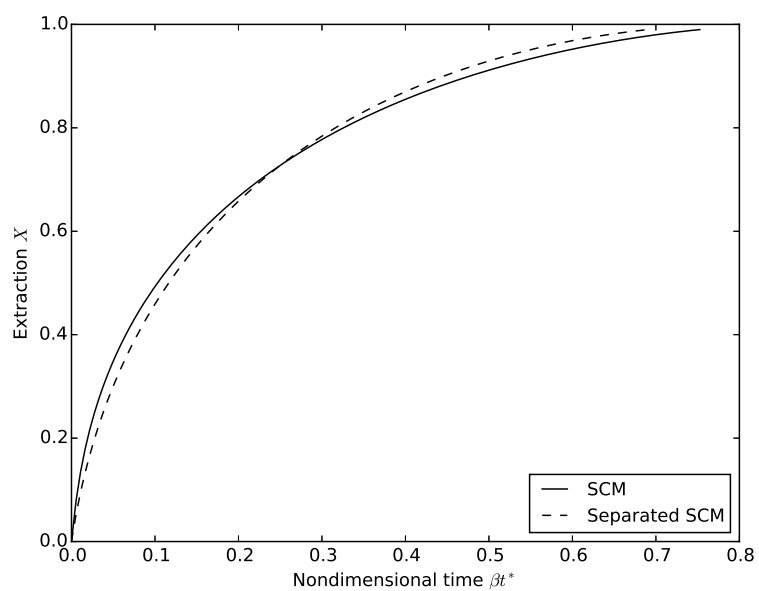

Figure 2: Extraction curves corresponding to $\kappa_{c}=10$, representing the worst fit

\section{A New Semi-Empirical Approach}

We may go on to generalise the notion of a separated model. The effect of the current state of the ore, characterised by, for instance, the extent of conversion $X$, may now be represented by some function $f(X)$. The dependency on external reagent concentration, $C_{\mathrm{ext}}^{n^{\prime}}$, can be extended to take into account other external conditions, such as temperature, $\mathrm{pH}$, Eh, wetting etc. The rate equation is now expressed as follows:

$$
\frac{\mathrm{d} X}{\mathrm{~d} t}=k_{\text {ext }}\left(C_{\text {ext } 1}, C_{\text {ext } 2}, T_{\text {ext }}, \mathrm{pH}, \mathrm{Eh}, \ldots\right) f(X)
$$

If the function $f$ is dimensionless, then the function $k_{\text {ext }}$ takes the form of a first order rate constant and has units of inverse time, although it is not a requirement of this method that $f$ be dimensionless. $k_{\text {ext }}$ depends on the concentration of chemical species in the fluid between the ore particles, as well as the temperature, $\mathrm{pH}$, Eh, etc. It will also depend on factors that influence the mass transfer between this bulk fluid and the particle surface, such as the liquid holdup or saturation, which affects the surface wetting. The most important thing is that the form of the relationship for $k_{\text {ext }}$ is correct as any constant prefactors can be absorbed into the function $f$, which is obtained empirically. $f$, while explicitly dependent on the extent of reaction, will be implicitly dependent on the size distribution of the particles, the size and spatial distribution of the mineral grains within the ore particles and the accessibility of the mineral grains due to the porosity and permeability of the ore particles.

It is important to note that this assumption of separability between the effect of the conditions experienced by the ore and the current extraction extent on the leaching rate is only meant to apply locally at the heap scale. That is, it applies to a region of ore that can be assumed to be experiencing the same conditions, not the entire ore body (in a continuum model this will typically be a differentially small region). This is because any system with non-linear dependencies will have an average behaviour that is different to the behaviour at the average 


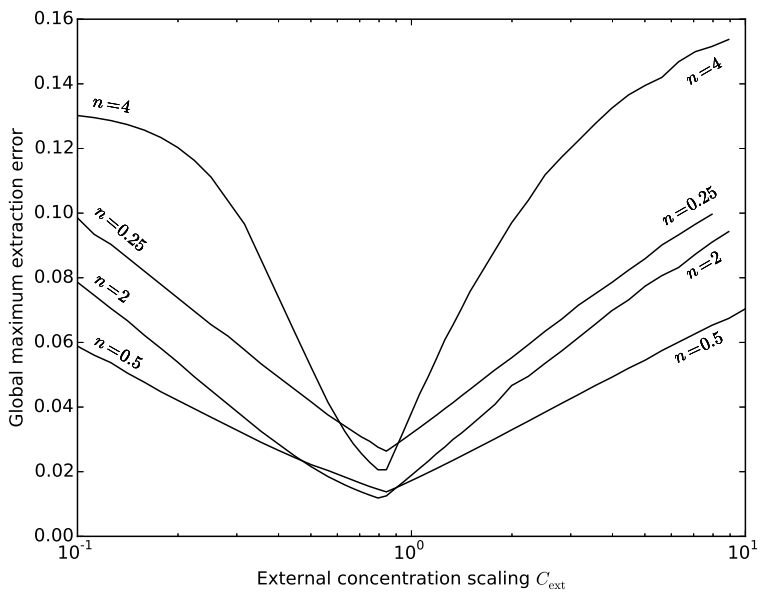

Figure 3: The peak maximum error analysed over a range of nondimensional external concentrations and selected intrinsic reaction orders

conditions, making modelling based purely on average conditions problematic. To model leaching behaviour, this model for the extraction will still need to be embedded within a system of models that describe the liquid flow, mass transport, heat balance etc.

In the presence of a particle size distribution, the proposed method would be valid as long as (i) the distribution remains invariant between the laboratory and the target application, or (ii) $f(X)$ is calibrated for a range of particle sizes, and from these a composite $f(X)$ is derived to suit the target particle size distribution. The method is likely to be valid anyway if the size of a particle cluster remains invariant, since the effective diffusion will be dominated by inter-particle diffusion. A notable advantage compared to the SCM is that calibration remains noniterative. A disadvantage is that scaling up to untested particle sizes would no longer be based on deterministic relationships and would therefore be ill-advised. However, we envisage that the particle sizes tested in the laboratory would be representative of those found in the target industrial setting.

\subsection{Theoretical basis for the separability assumption in the context of heap leaching}

In $\S 3$, the assumption that the effect of the external conditions on the leaching rate is separable from that of the current state of the ore particles was shown to hold over a wide parameter space in the context of the shrinking core model. Our proposed method shares the same assumption, but additionally we need to assess the validity of the assumption at the three different scales of heap leaching.

The leaching behaviour needs to be considered at the scale of the mineral grains, individual ore particles and clusters of ore particles. For any mineral surface, the rate of reaction per surface area will be a function of the concentration of reagents and products adjacent to the surface and the temperature. The rate of dissolution of the mineral grain can thus be expressed as follows:

$$
\frac{\mathrm{d} m_{i}}{\mathrm{~d} t}=-k_{m s}\left(C_{1}, C_{2}, T, \ldots\right) A_{i}\left(m_{i}\right),
$$

where $m_{i}$ is the mass of grain $i, A_{i}$ is its surface area and $k_{m s}$ is the leaching rate per unit of surface area, which will typically have a form of, for example,

$$
k_{m s}=k_{m s_{0}}(T) C_{1}^{n_{1}} C_{2}^{n_{2}} .
$$

The concentrations and temperatures in Eq. (24) are those experienced by the mineral surface.

According to Equation (23), it is possible to separate the dependency of the grain shrinkage rate on (i) the conditions the grain is experiencing and (ii) the current state of the grain as manifested by its current size and surface area. Therefore, behaviour is separable at the level of an individual mineral grain.

Within an ore particle the different mineral grains will experience different chemical conditions depending upon their location within the particle. If, though, we consider a subset of $N$ grains which are experiencing similar chemical conditions (e.g. within a small region of the ore particle), then the total leaching rate of these grains is

$$
\frac{\mathrm{d} m_{\mathrm{tot}}}{\mathrm{d} t}=-k_{m s}\left(C_{1}, C_{2}, T, \ldots\right) \sum_{i=1}^{N} A_{i}\left(m_{i}\right)
$$


The summation term depends only on the current state of all the mineral grains being considered. It can also be shown that this current state is uniquely defined by $m_{\text {tot }}$ (see Appendix A). We can therefore write

$$
\frac{\mathrm{d} m_{\mathrm{tot}}}{\mathrm{d} t}=-k_{m s}\left(C_{1}, C_{2}, T, \ldots\right) A\left(m_{\mathrm{tot}}\right)
$$

which means that the behaviour of a set of grains experiencing the same chemical conditions is separable irrespective of, for instance, the size distribution of those grains.

The chemical and other conditions within an ore particle will vary both spatially and temporally. This implies that $k_{m s}$ within an ore particle will also vary with both space and time. The behaviour of the entire ore particle will only be separable if the value of $k_{m s}$ at a particular position is uniquely defined by the value in the surrounding fluid $k_{\text {ext }}$ and the current average extent of reaction for that ore particle. This requirement can be expressed as follows:

$$
k_{m s}(\mathbf{r})=k_{\text {ext }} g(\mathbf{r}, X)
$$

where $\mathbf{r}$ is the position within the ore particle.

This requirement is only true under certain sets of assumptions. The first assumption under which it will be true is if the system is reaction-controlled (fast diffusion) as this will result in a constant set of chemical conditions within the ore particle. For most heap leaching situation this is unlikely to be true as diffusion through the ore particle is usually a significant factor in the overall rate.

This will also be true if the ore particle can be assumed to be at pseudo-steady state and the surface reaction kinetics are linear. The pseudo-steady state assumption implies that the accumulation terms associated with changes in the concentration within the fluid are negligible compared to the fluxes of the chemical species through the particle. This is a reasonable assumption and one that is shared with the shrinking core model. An assumption that the surface reactions are all linear, together with the fact that diffusion is also a linear process means that all the rates within the system can be scaled by the external concentrations, which means that Equation (27) holds.

A final situation in which the behaviour is separable is if the reactions are completely diffusion-controlled. Under these conditions the concentration of the limiting reactant at the mineral surface will be zero and the reaction rate will be dependent only on the driving force for the diffusion, which will be proportional to the concentration of this reactant in the surrounding fluid.

This means that the behaviour is not likely to be completely separable if the surface reactions rates are very non-linear and neither the diffusion nor the surface reactions are rate limiting. Non-separability implies that there is a history dependence to the reaction rate that is not captured by the average extent of reaction. For instance, in a non-separable system if two identical ore particles were initially subjected to different chemical and/or environmental conditions, but arrived at the same average final state (e.g. same overall extraction), the leaching rates of these particles would be different even if subsequently subjected to identical chemical and environmental conditions, while the rates would be the same for a separable system as its behaviour is uniquely defined by the current state.

As many real heap leaching system have nonlinear surface reaction kinetics and are not necessarily completely reaction- nor diffusion-controlled, the real system behaviour may not be completely separable. This does not mean that this assumption is not a useful one as it makes the problem much more mathematically tractable and, as demonstrated in $§ 3.1$, the history dependence is still likely to be much weaker than the dependency on the average current state. This is especially true if the conditions used in the calibration tests are similar to those experienced by the system being predicted.

Finally, if the behaviour of a single ore particle is mathematically separable, then the behaviour of a collection of different ore particles all experiencing the same chemical conditions will be separable for identical reasons to why a set of individual grains experiencing the same chemical conditions are separable. This separability holds irrespective of, for instance, the size distribution of the ore particles or the distribution of the mineral grains within the ore particles.

\subsection{Calibrating the model}

When using the shrinking core model with a distribution of particle sizes or rate constants, the parameters in the model need to be iteratively adjusted to obtain a good fit between the model and the calibration data. In the present approach, as long as the extraction and the chemical conditions experienced by the ore are known, the model can be calibrated without any iteration.

Equation (22) can be rearranged to the following form:

$$
f(X)=\frac{1}{k_{\text {ext }}} \frac{\mathrm{d} X}{\mathrm{~d} t} .
$$


We assume that the form for $k_{\text {ext }}$ is either known from additional experiments or synthesised from an appropriate deterministic model. The latter approach is used in this work for the purpose of validation, and is explained in §5.3. In the calibration tests the extraction as a function of time is known, which means that the rate of change of extraction with time can also be obtained either directly by numerical differentiation or, if the data is noisy, by fitting a curve to the extraction data and differentiating the fitted curve.

As the conditions (chemical concentrations, temperature etc.) experienced by the ore particles in the calibration experiments are assumed to be known, the value of $k_{\text {ext }}$ that corresponds to each value of can be calculated. This is easily done with small column leaching experiments in which spatial variations are small. The function $f$ can thus be obtained by plotting the rate of $X$, divided by $k_{\text {ext }}$, against $X$.

This relationship can then be used in simulations of heap performance using either a lookup table with interpolation between the known points or by fitting a curve to this relationship. It must of course be coupled to appropriate models for the fluid flow, mass transport and heat transport within the heap.

\section{Calibration and Validation Example}

Data from column leaching experiments may be used to calibrate and/or validate the semi-empirical model. A comprehensive validation would include multiple sets of data corresponding to variations in reagent concentration, temperature, $\mathrm{pH}$ and so on, in order to show that Equation (22) holds. In practice, however, the design and execution of a comprehensive set of experiments is not trivial given the long leaching times (of the order of several months). For this paper, a single case study was carried out based on two sets of data. One set of data was used to calibrate the model; the other was used to test the model's predictive capability in the presence of a differing external reagent concentration. The first set of data features in Cai et al. (2014).

Three parametrisation models were also calibrated and tested against the data. It is interesting to compare the performance of these models, but there is another motive for using them in this work. As discussed in the previous section, the form of $k_{\text {ext }}$ must be known before calibrating the semi-empirical model. We may assume, as we effectively did for the separated SCM in $\S 3$, that

$$
k_{\text {ext }}=C_{\text {ext }}^{n^{\prime}} \text {. }
$$

However, it is not possible to know the apparent order of reaction $n^{\prime}$ in advance. We may predict that it is approximately 0.42 if the system is reaction-controlled (Kimball et al., 2010; Madsen and Wadsworth, 1980) or unity if it is diffusion-controlled, but we still do not know the regime in the first place.

The easiest way to choose $n^{\prime}$ is to have a third set of data against which the parameter may be optimised. In the absence of such data, however, one solution to the problem is to find a parametrisation model which can be optimised to fit the calibration data and which scales correctly to fit the validation data. We may infer from the model's optimum reaction modulus which regime the system is under. If the regime is intermediate, then in the spirit of the analysis in $\S 3.1$ the parametrisation model may be separated and $n^{\prime}$ tuned to minimise the least squares error between the curves. This was the approach adopted for the present paper.

Because the columns were small and an average reagent concentration could be inferred from the feed value and time series measurements at the outlet, it was possible to test the particle scale models directly without coupling to a column scale simulator. The only assumption that needed to be made was the shape of the reagent concentration profile within the column, since this affects the average value at a given time. The approach taken was to assume a quadratic profile which was constrained by the inlet and outlet values and a zero gradient at the outlet, as shown in Figure 4. The models were run again with different profile shapes to check sensitivity. As Appendix B shows, the numerical results are not very sensitive to the assumed concentration profile.

Particles and clusters were assumed to be of a constant size, which is a reasonable assumption given that the particle size distribution was narrow. As discussed previously, unless the cluster scale dominates diffusion and cluster size remains invariant, a wide particle size distribution complicates the modelling and is beyond the scope of the present paper. In particular, the size distribution affects the shrinking core and progressive conversion models and the estimation of their associated reaction moduli. It is worth noting, however, that it will not have a direct impact on the calibration of $f(X)$ once the form of $k_{\text {ext }}$ has been decided.

\subsection{Experimental Method}

Leaching experiments were carried out in a small laboratory scale column of height $190 \mathrm{~mm}$ and internal diameter $28 \mathrm{~mm}$. The ore was a copper sulphide ore from the Rio Tinto Kennecott mine, Utah. The particle diameters ranged from $8 \mathrm{~mm}$ to $11.2 \mathrm{~mm}$. While such a large particle size in relation to the column diameter may have induced a disproportionate fraction of the reagent channelling between the column walls and ore particles, it also meant that the external concentrations experienced by all the particles were similar. The particle surfaces remained fully wetted and, as noted above, there was not a high sensitivity to spatial variations in the external reagent concentration. However, we accept that such wall effects would need to be taken into account in a column- or heap-scale simulation.

The majority of the copper was in the form of chalcopyrite with average initial copper grade of $0.7 \%-0.8 \%$. The 


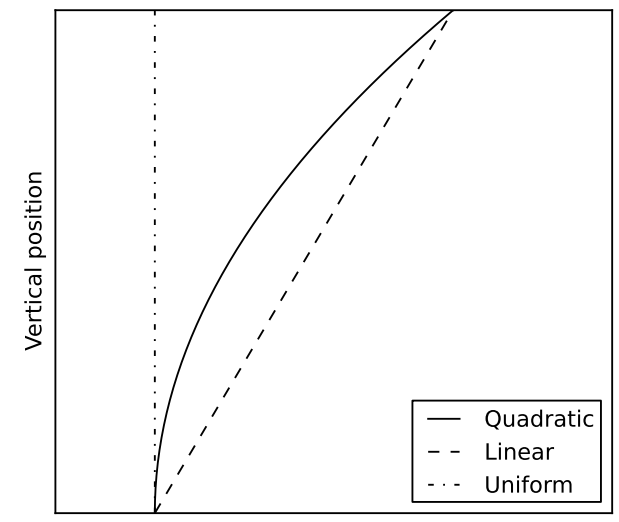

Relative external reagent concentration $C_{\text {ext }}$

Figure 4: Possible concentration profiles. The quadratic profile was assumed for analysis.

other major sulphide component of the ore was pyrite. From a mineral volume perspective, the amount of iron was much greater than that of copper, contained in pyrite (4.5 vol\%) and chalcopyrite (0.5 vol\%), with minor amounts of covellite, chalcocite and other copper-bearing phases.

In real leaching systems, the oxidising agents are ferric iron, which is converted from ferrous by naturally occurring bacteria, and dissolved oxygen. In the experiments, ferric sulphate was added to the feed at a controlled redox potential such that ferric iron could be considered the dominant reagent. The following reaction takes place (Madsen and Wadsworth, 1980):

$$
\mathrm{CuFeS}_{2}+4 \mathrm{Fe}^{3+} \rightarrow \mathrm{Cu}^{2+}+5 \mathrm{Fe}^{2+}+2 \mathrm{~S}^{0} .
$$

The feed $\mathrm{pH}$ was held at 1.2 using $0.1 \mathrm{M}$ sulphuric acid, preventing the formation of jarosite which has an otherwise inhibiting effect on the reaction rate. We further assume that all the elemental sulphur generated from oxidation of chalcopyrite was further oxidised so that no elemental sulphur accumulated.

In both experiments, ferric sulphate was added to the feed at a concentration of $5 \mathrm{~g} / \mathrm{L}$ (5000 ppm). The experiments differed only in the redox potential of the feed and hence the concentration of ferric iron present. These conditions are are listed in Table 1.

Table 1: Feed conditions for the two experiments

\begin{tabular}{ccc}
\hline Experiment & \multicolumn{2}{c}{ Concentration } \\
& Ferric $\left[\mathrm{Fe}^{3+}\right]$ & Ferrous $\left[\mathrm{Fe}^{2+}\right]$ \\
\hline \multirow{2}{*}{$\mathrm{A}$} & $5 \mathrm{~g} / \mathrm{L}$ & nil \\
& $(89.3 \mathrm{mM})$ & $4 \mathrm{~g} / \mathrm{L}$ \\
\multirow{2}{*}{$\mathrm{B}$} & $1 \mathrm{~g} / \mathrm{L}$ & $(71.4 \mathrm{mM})$ \\
\hline
\end{tabular}

The extraction was monitored by periodically measuring the volume of effluent from the column and the concentrations of copper and both ferric and ferrous iron species in the solution. Plots of ferric concentration, which was fed into the models, can be found in Appendix B. In addition to the concentrations of the metal species, the $\mathrm{pH}$ and $\mathrm{Eh}$ of the solution were also measured. To minimise the effects of temperature variation on the reaction rates, the columns were kept in a temperature-controlled housing maintained at $60^{\circ} \mathrm{C}$. The flow rate into the column was maintained at $82.7 \mu \mathrm{L} / \mathrm{min}\left(8.06 \mathrm{~L} / \mathrm{m}^{2} / \mathrm{hr}\right)$ to ensure a constant saturation.

In addition to the chemical measurements, the columns were scanned using X-ray micro tomography (XMT) at regular intervals. This gave measurements for the total sulphide extraction. The ore particles were scanned using a Nikon Metris Custom Bay with a $1 \mathrm{~mm}$ aluminium filter to reduce the effect of beam hardening, $89 \mathrm{kV}$ energy, $0.708 \mathrm{~s}$ exposure time and 2001 projections. The detector size was $2000 \times 2000$ pixels, giving a linear resolution of approximately $17 \mu \mathrm{m}$ for the magnification selected (Lin et al., 2015).

In accordance with Lin et al. (2015), the procedure for the image processing was as follows:

1. A $3 \times 3 \times 3$ median filter was applied to the reconstructed image to reduce the noise level.

2. The threshold for distinguishing the ore particles from the air phase was obtained using the Otsu algorithm (Otsu, 1975) 
3. The metal sulphide grains were distinguished from the ore matrix using a maximum entropy algorithm (Kapur et al., 1985).

The reason for using the different algorithms was that the air and rock have very distinct peaks in the intensity histogram, while the relatively small volume of metal sulphide present means that there is no distinct peak in the histogram. Figure 5 provides visualisations before and after leaching.

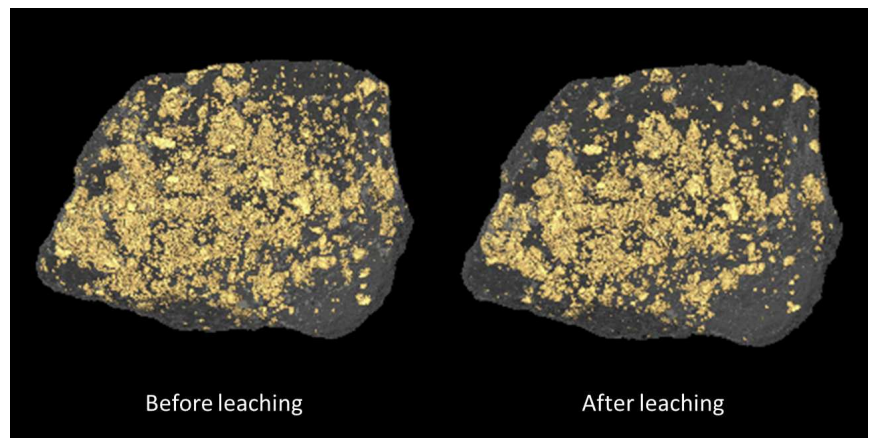

Figure 5: Micro-CT scan of a single rock, showing the state of the mineral grains before and after leaching

Assuming that the sulphide consisted only of chalcopyrite and pyrite and combining the copper extraction based on the chemical analysis of the effluent with these XMT measurements allowed the extraction of pyrite to be estimated.

\subsection{Existing Models}

The models shortlisted for testing were the standard shrinking core model, the two-layer shrinking core model of Velardo et al. (2002) and the progressive conversion model of Dixon and Hendrix (1993a). The latter model was implemented with second order finite differences; 41 points was found to be a sufficient resolution. The PCM incorporating the extended K- $\varphi$ model (Ghorbani et al., 2013) was not considered, since it is impossible to isolate $t$ in Equation (18) and hence reconstruct a rate equation that could be practically used in a heap leaching simulator.

In parametrising these models, it was assumed that the extent of leaching could be expressed by Equation (30), i.e. the fraction of chalcopyrite leached was equal to the fraction of copper leached.

\subsubsection{Optimisation Method}

The models were passed to an optimiser that adjusted the parameters to minimise the least squares error between the resulting extraction curve and the experimental data. The DAE solver in Assimulo (Andersson et al., 2014) was used to integrate the extraction curves. Given the nonlinear, multi-parameter nature of the optimisation, it was important to use a global minimisation algorithm in conjunction with a local minimiser. We used the basin-hopping algorithm implemented in SciPy (Jones et al., 2001), running it several times to check that the results were consistent. To allow bounds to be placed on the parameters, the L-BFGS-B algorithm was chosen as the local minimiser.

For the two SCMs, the pseudo-steady state assumption could be made because $\beta$ from Equation (2) was estimated to be small. Specifically, with an estimated ore density of $2700 \mathrm{~kg} / \mathrm{m}^{3}$, porosity of $3-5 \%$, and the maximum ferric concentration and mineral grade given in the previous section, it was estimated that $2.3 \times 10^{-3} \leq \beta \leq 4.0 \times 10^{-3}$. To simplify optimisation of the PCM without reformulating the model as pseudo-steady, $\beta$ was given a fixed value of $4.0 \times 10^{-3}$ and $t_{D} / \beta$ was allowed to vary.

In all cases, it was desirable to place bounds on the model parameters before they were passed to the optimiser so that they did not arrive at nonphysical values. These bounds are summarised in Table 2. No assumption was made about bounds on the rescaled diffusion time $t_{D} / \beta$, reaction moduli $\kappa_{c}$ and $\kappa_{p}$ and shape parameter $\alpha_{2}$, so these were made arbitrarily high and low. In the absence of further information, arbitrary upper bounds were placed on the reaction rate scale factor $\alpha_{1}$ in the two-layer model and on the proportion of surface mineral grains $\lambda$ in the Dixon-Hendrix model.

According to Dixon and Hendrix (1993b), The mineral order of reaction $m_{p}$ is $2 / 3$ when the mineral grains are distributed throughout the solid as uniform spheres. In reality, of course, the mineral grains will have different shapes and sizes. Dixon and Hendrix suggest that a log-normal distribution of mineral grain sizes may be approximated by raising the value of $m_{p}$. For example, a standard deviation of 1 corresponds to $m_{p} \approx 1.5$ and a standard deviation of 2 corresponds to $m_{p} \approx 3.5$. Therefore $m_{p}$ was made an optimisable parameter and given appropriate bounds.

After the models were optimised using data from Experiment A, they were run again using ferric concentration 
Table 2: Parameters to be optimised

\begin{tabular}{ccc}
\hline Parameter (units) & \multicolumn{2}{c}{ Bounds } \\
\hline All models & & \\
$t_{D} / \beta$ (days) & 1 & $10^{5}$ \\
$\kappa_{c}, \kappa_{p}$ & $10^{-3}$ & $10^{3}$ \\
\hline Two-layer & & \\
$\alpha_{1}$ & 1 & 10 \\
$\alpha_{2}$ & $10^{-2}$ & $10^{2}$ \\
$\xi_{i}$ & 0.5 & 1.0 \\
\hline Dixon-Hendrix & & \\
$m_{p}$ & $2 / 3$ & 3 \\
$\lambda$ & 0 & 0.25 \\
\hline
\end{tabular}

measurements from Experiment B. The resulting extraction curves could then be compared against Experiment B's extraction data.

\subsubsection{Results}

The optimum parameters are given in Table 3, with the associated extraction curves shown in Figure 6. Scaling to Experiment B resulted in the extraction curves shown in Figure 7.

Table 3: Parameter values following optimisation

\begin{tabular}{lccc}
\hline Parameter (units) & \multicolumn{3}{c}{ Optimum values } \\
& SCM & TLSCM & DHPCM \\
\hline$t_{D} / \beta$ (days) & 1770 & 15.5 & 5.65 \\
$\kappa_{c}, \kappa_{p}$ & 660 & 0.00384 & 0.222 \\
\hline$\alpha_{1}$ & - & 10.0 & - \\
$\alpha_{2}$ & - & 3.23 & - \\
$\xi_{i}$ & - & 0.764 & - \\
\hline$m_{p}$ & - & - & 2.11 \\
$\lambda$ & - & - & 0.01 \\
\hline
\end{tabular}

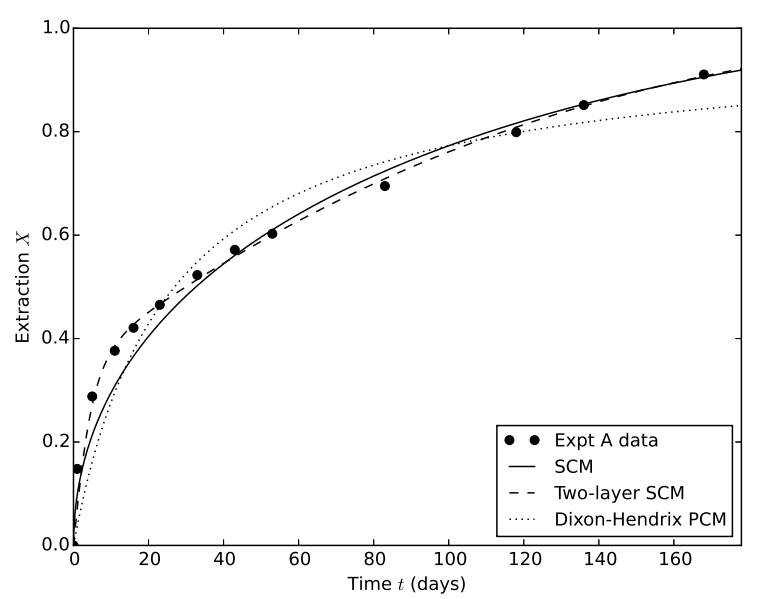

Figure 6: Model extraction curves, optimised to fit data from Experiment A

\subsubsection{Discussion}

All of the models were optimised to have a low reaction modulus, except for the SCM which became diffusioncontrolled. However, the SCM scales poorly to the Experiment B data. This highlights the common danger of curve fitting: a good initial fit does not necessarily mean we have arrived at realistic parameters, or even that we are using the correct model. The SCM in particular is often parametrised against reference data by curve fitting or, more simply, by plotting $1-(1-X)^{1 / 3}$ and $1-3(1-X)^{2 / 3}+2(1-X)$ against time. These latter expressions come about from analytical integration of the SCM equation in limiting cases, c.f. Equation (21). The idea is that if the data can be shown to follow a straight line on one of these plots, it indicates the regime is reaction-controlled or diffusion-controlled respectively. As Liddell observed, however, it is often not too difficult to fit a straight line to either plot. 


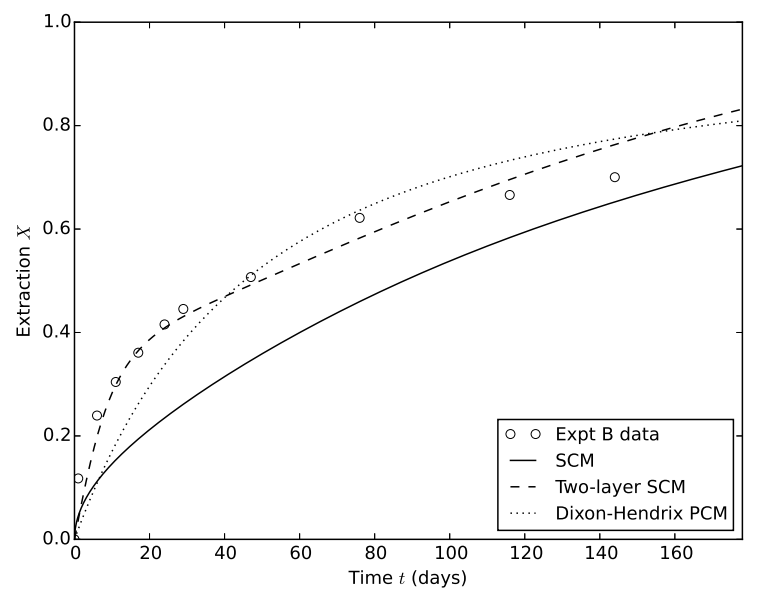

Figure 7: Model extraction curves, scaled according to Experiment B conditions and compared against the corresponding extraction data

By contrast, the two-layer SCM does a good job of capturing the knee at $X \approx 0.4$ in both sets of data. It is intuitive that having a step function built into the model would enable it to capture such features, but the ability of the model to scale correctly to the validation data suggests that the correct regime is represented. A point of concern is that the reaction rate scale factor $\alpha_{1}$ took the maximum allowable value of 10 . To say whether or not this is physically realistic would require further investigation.

The PCM does not fit the data very well. Despite appearing to support a reaction-controlled regime, in the sense that $t_{D} / \beta$ was optimised to a value somewhere between the specified bounds, the rate becomes very slow towards the end of leaching. The surface parameter $\lambda$ has been optimised out. This is perhaps unsurprising since, given that the other surface parameters were fixed at $\kappa_{s}=\frac{\lambda}{1-\lambda} \kappa_{p}$ and $m_{s}=m_{p}$, increasing $\lambda$ will only make the system more reaction-controlled. It is speculated that the model could perform better if $\kappa_{s}$ and $m_{s}$ were allowed to vary.

\subsection{Proposed Model}

The previous section established the two-layer SCM as an exemplary model that scales correctly to the validation data. This model can be used to establish a dependency on external reagent concentration $k_{e x t}=C_{e x t}^{n^{\prime}}$ which is needed before we can calibrate the proposed model using experimental data.

We can separate the two-layer SCM equation just as we did with the standard SCM in $\S 3$.

$$
\frac{1}{\beta} \frac{\mathrm{d} \xi_{c}}{\mathrm{~d} t^{*}}=\frac{C_{\mathrm{ext}}^{n^{\prime}}}{1 / \kappa_{c}^{\prime}+\xi_{c}\left(1-\xi_{c}\right)} .
$$

The only difference between this and Equation (20) is $\kappa_{c}^{\prime}$, which is the two-layer rate modification given by Equation (10).

The apparent order of reaction $n^{\prime}$ was tuned once again so that integration of Equation (31) matched the twolayer SCM extraction curve in Figure 7 as closely as possible. Unsurprisingly, given the low reaction modulus, $n^{\prime}$ was tuned to the intrinsic order $n$ with no visible error between the curves.

\subsubsection{Calibration}

Calibration of the method involved fitting a curve to the experimental data before numerically differentiating the curve. Construction of the curve was complicated somewhat by the fact that the experimental data was sparse and noisy. For this purpose, a curve-fitting function was adapted from the Gridfit MATLAB function by d'Errico (2005). Gridfit builds 2D surfaces from scattered data, minimizing the distance between the surface and the data points in the least squares sense. Because the system of equations is generally underdetermined, a regulariser is added to provide smoothness.

We translated Gridfit to 1D, implementing the Laplacian regulariser (i.e. minimisation of the curvature of the line) and modifying the regulariser to have less weight in the earlier stages of leaching, where it was more important to preserve the sharpness of the curve, and more weight in the later stages where smoothness was important. The data points were also weighted so that the curve could be forced to fit the earlier data more closely. The resulting curve was differentiated using second order differences and divided by $k_{\text {ext }}$ associated with Experiment A. $k_{\text {ext }}$ in this instance was approximately unity, since the reagent concentration at outlet did not vary much from the inlet value for the duration of the experiment. The final function is shown in Figure 8 . 
When the model was integrated in time according to the reference reagent concentration, the previously fitted curve was naturally recovered (to within the discretisation errors associated with the second order differences and the ODE solver). Figure 9 shows this curve along with the two-layer SCM curve for comparison.

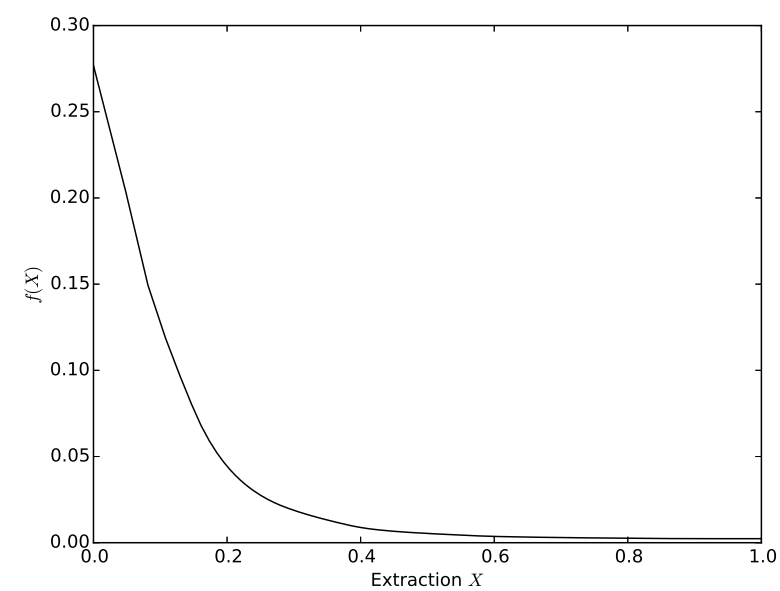

Figure 8: The function $f(X)$ as calibrated from Experiment A data

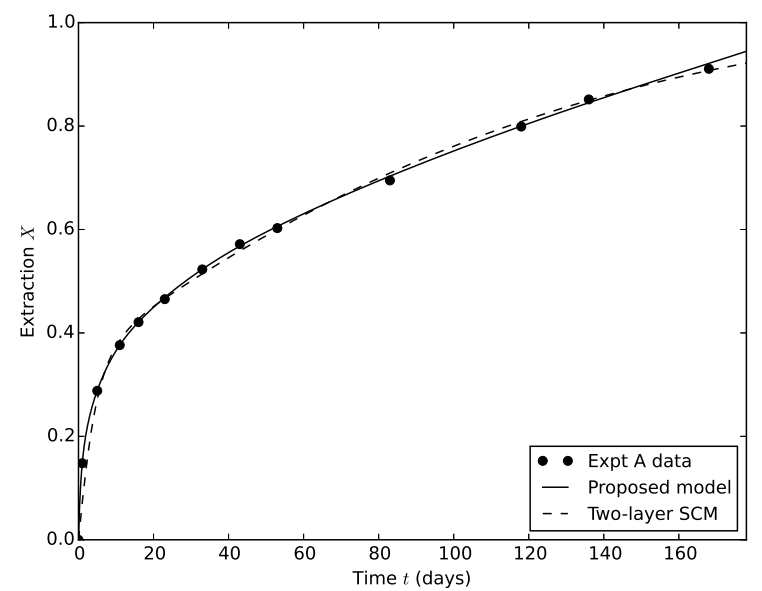

Figure 9: Proposed model extraction curve after calibration (which appears identical to the fitted curve due to numerical differentiation and integration) with the two-layer SCM for comparison

\subsubsection{Validation}

When the model was integrated in time according to the Experiment B reagent concentration history, the curve in Figure 10 was produced. It can be seen that the proposed model fits the data well and shows close agreement with the two-layer SCM.

This favourable performance of the proposed model lends the model credibility, although it is difficult to comment further on how the model would scale to other conditions. More testing is deferred to future work.

\section{Conclusions}

The main findings of the present study may be summarised as follows:

- In the presence of an intermediate control regime with nonlinear surface kinetics, it is possible to replace the implicit formulation of the shrinking core model with a more economical explicit formulation and still retain accuracy. This may be of interest to researchers wishing to couple the SCM to a heap scale simulator under such conditions.

- The two-layer SCM of Velardo et al. (2002) appears to perform well in our case study compared to the standard SCM and the progressive conversion model of Dixon and Hendrix (1993a). 


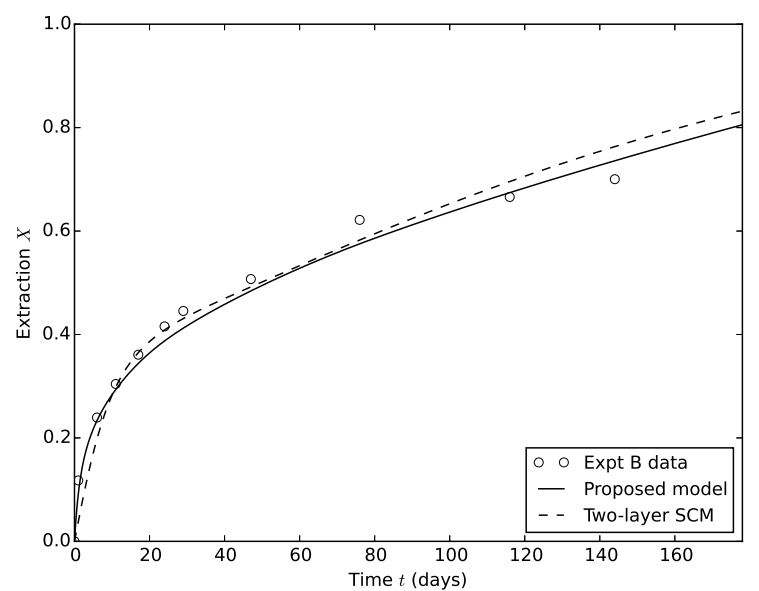

Figure 10: Proposed model extraction curves, scaled according to Experiment B conditions and compared against the corresponding extraction data, with the two-layer SCM for comparison

- Our proposed semi-empirical model also performs well and may be of interest to the heap leaching community as an economical, easy-to-calibrate alternative to the SCM or PCM.

We accept that a single case study is unlikely to establish the proposed model as the preferred tool for industrial use. Comprehensive validation of the method will require more test data from suitably controlled experiments and is left for future work.

\section{Appendix A}

Proof that, given $\mathrm{d} m_{i} / \mathrm{d} t=-k_{m s} A_{i}\left(m_{i}\right)$ and $m_{\mathrm{tot}}=\sum_{i} m_{i}$, there exists a function $A$ such that $\mathrm{d} m_{\text {tot }} / \mathrm{d} t=-k_{m s} A\left(m_{\text {tot }}\right)$.

$$
\frac{\mathrm{d} m_{\mathrm{i}}}{\mathrm{d} t}=-k_{m s} A_{i}\left(m_{i}\right),
$$

therefore by chain rule

$$
\frac{\mathrm{d} m_{\mathrm{i}}}{\mathrm{d} X} \frac{\mathrm{d} X}{\mathrm{~d} t}=-k_{m s} A_{i}\left(m_{i}\right)
$$

If $X$ is chosen such that $\mathrm{d} m_{i} / \mathrm{d} t=-k_{m s}$ (or, equivalently, $X=-\int_{0}^{t} k_{m s} \mathrm{~d} t$ ), then

$$
\frac{\mathrm{d} m_{i}}{\mathrm{~d} X}=A_{i}\left(m_{i}\right)
$$

This implies that

$$
m_{i}=f_{i}(X)
$$

Since $m_{\mathrm{tot}}=\sum_{i} m_{i}$ this implies that $\mathrm{d} m_{i} / \mathrm{d} t=-k_{m s} \sum_{i} A_{i}\left(m_{i}\right)$. Therefore

$$
\begin{aligned}
\mathrm{d} m_{\text {tot }} / \mathrm{d} X & =\sum_{i} A_{i}\left(m_{i}\right) \\
& =\sum_{i} A_{i}\left(f_{i}(X)\right) .
\end{aligned}
$$

Therefore $m_{\mathrm{tot}}=f(X)$.

Since $m_{\text {tot }}$ and all the $m_{i}$ values are functions of $X$ only, this implies that

$$
\sum_{i} A_{i}\left(f_{i}(X)\right)=A(f(X)),
$$

which means that there exists a function $A$ such that

$$
\frac{\mathrm{d} m_{\mathrm{tot}}}{\mathrm{d} t}=-k_{m s} \sum_{i} A_{i}\left(m_{i}\right)=-k_{m s} A\left(m_{\mathrm{tot}}\right) .
$$




\section{Appendix B}

Figure 11 shows the measured ferric concentrations at outlet for the two experiments. The fitted lines were used in conjunction with the feed concentrations (Table 1) and an assumed profile shape in order to calculate the average reagent concentration. The form of the fit with respect to time in Figure 11 has very little impact on the validation curves, especially for the better-fitting models. When Figure 7 is regenerated using the uniform or linear concentration profiles, the errors in $X$ do not vary from the quadratic concentration by more than 0.066 for the SCM, 0.017 for the the Dixon-Hendrix model and 0.029 for the two-layer SCM.

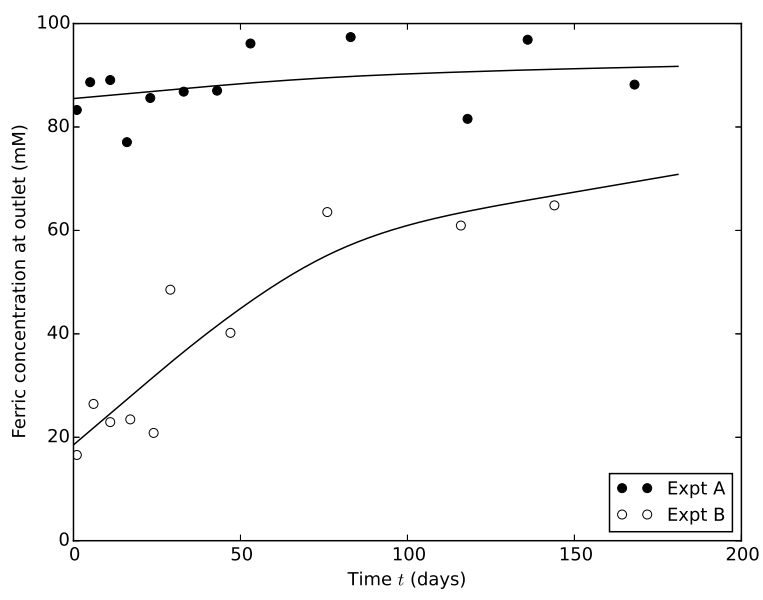

Figure 11: Outlet ferric concentration histories

\section{Acknowledgements}

The experiments were carried out in the Rio Tinto Centre for Advanced Mineral Recovery at Imperial College London. The authors gratefully acknowledge financial support from Rio Tinto.

The authors thank the anonymous reviewers for their constructive comments. The first author would like to thank Steven Dargaville of the Applied Modelling and Computation Group for his advice on modelling moving boundary problems.

\section{References}

C. Andersson, C. Führer, and J. Åkesson. Assimulo: A unified framework for ODE solvers. Technical Report LUTFNA-5005-2014, Centre for Mathematical Sciences, Lund University, 2014.

R. W. Bartlett. A combined pore diffusion and chalcopyrite dissolution kinetics model for in situ leaching of a fragmented copper porphyry. In Proceedings of International Symposium on Hydrometallurgy, pages 331-374, 1973.

R. W. Bartlett. Simulation of ore heap leaching using deterministic models. Hydrometallurgy, 29(1):231-260, 1992.

C. R. Bennett, D. McBride, M. Cross, and J. E. Gebhardt. A comprehensive model for copper sulphide heap leaching: Part 1 basic formulation and validation through column test simulation. Hydrometallurgy, 127: 150-161, 2012.

S. C. Bouffard and D. G. Dixon. Investigative study into the hydrodynamics of heap leaching processes. Metallurgical and Materials Transactions B, 32(5):763-776, 2001.

R. L. Braun, A. E. Lewis, and M. E. Wadsworth. In-place leaching of primary sulfide ores: laboratory leaching data and kinetics model. Metallurgical Transactions, 5(8):1717-1726, 1974.

L. Cai, R. J. Ferrier, Q. Lin, P. Mostaghimi, G. J. Gorman, and S. J. Neethling. Heap leaching simulation: Beyond shrinking core models. In Proceedings of 10th International Conference on CFD in Oil and Gas, Metallurgical and Process Industries, 2014.

M. Cross, C. R. Bennett, T. N. Croft, D. McBride, and J. E. Gebhardt. Computational modeling of reactive multi-phase flows in porous media: Applications to metals extraction and environmental recovery processes. Minerals Engineering, 19(10):1098-1108, 2006. 
J. d'Errico. Gridfit: Model 2-d surfaces from scattered data (http://uk.mathworks.com/matlabcentral/fileexchange/8998surface-fitting-using-gridfit). MATLAB Central File Exchange, 2005. [Online; accessed 2015-02-26].

D. G. Dixon. Analysis of heat conservation during copper sulphide heap leaching. Hydrometallurgy, 58(1):27-41, 2000 .

D. G. Dixon and J. L. Hendrix. A general model for leaching of one or more solid reactants from porous ore particles. Metallurgical Transactions B, 24(1):157-169, 1993a.

D. G. Dixon and J. L. Hendrix. Theoretical basis for variable order assumption in the kinetics of leaching of discrete grains. AIChE journal, 39(5):904-907, 1993b.

Y. Ghorbani, M. Becker, A. Mainza, J.-P. Franzidis, and J. Petersen. Large particle effects in chemical/biochemical heap leach processes - a review. Minerals Engineering, 24(11):1172-1184, 2011.

Y. Ghorbani, J. Petersen, M. Becker, A. N. Mainza, and J.-P. Franzidis. Investigation and modelling of the progression of zinc leaching from large sphalerite ore particles. Hydrometallurgy, 131:8-23, 2013.

E. Jones, T. Oliphant, P. Peterson, et al. SciPy: Open source scientific tools for Python (http://www.scipy.org), 2001. [Online; accessed 2015-02-26].

J. N. Kapur, P. K. Sahoo, and A. K. Wong. A new method for gray-level picture thresholding using the entropy of the histogram. Computer vision, graphics, and image processing, 29(3):273-285, 1985.

B. E. Kimball, J. D. Rimstidt, and S. L. Brantley. Chalcopyrite dissolution rate laws. Applied Geochemistry, 25 (7):972-983, 2010 .

M. Leahy, M. Davidson, and M. Schwarz. A model for heap bioleaching of chalcocite with heat balance: Bacterial temperature dependence. Minerals Engineering, 18(13-14):1239-1252, 2005. ISSN 0892-6875. doi: 10.1016/j.mineng.2005.07.003.

M. J. Leahy, M. R. Davidson, and M. P. Schwarz. A two-dimensional model for the heap bioleaching of chalcocite: effect of inlet height. In Fifth International Conference on CFD in the Process Industries, 2006.

O. Levenspiel. Chemical Reaction Engineering, volume 2. Wiley New York etc., 1972.

K. C. Liddell. Shrinking core models in hydrometallurgy: What students are not being told about the pseudosteady approximation. Hydrometallurgy, 79(1):62-68, 2005.

Q. Lin, S. J. Neethling, K. J. Dobson, L. Courtois, and P. D. Lee. Quantifying and minimising systematic and random errors in X-ray micro tomography based volume measurements. Computers \& Geosciences, 2015.

B. W. Madsen and M. E. Wadsworth. A mixed kinetics dump leaching model for ores containing a variety of copper sulfide minerals. U. S. Dept. of Interior, pages 1-44, 1980.

D. McBride, M. Cross, and J. Gebhardt. Heap leach modeling employing CFD technology: A 'process' heap model. Minerals Engineering, 33(0):72-79, $2012 . \quad$ ISSN $0892-6875 . \quad$ doi: http://dx.doi.org/10.1016/j.mineng.2011.10.003.

G. Miller. Ore geotechnical effects on copper heap leach kinetics. Young, C A, et al. (Ed.), Hydrometallurgy, pages 329-342, 2003.

P. B. Munoz, J. D. Miller, and M. E. Wadsworth. Reaction mechanism for the acid ferric sulfate leaching of chalcopyrite. Metallurgical Transactions B, 10(2):149-158, 1979.

H. J. Neuburg, J. A. Castillo, M. N. Herrera, J. V. Wiertz, T. Vargas, and R. Badilla-Ohlbaum. A model for the bacterial leaching of copper sulfide ores in pilot-scale columns. International Journal of Mineral Processing, 31(3):247-264, 1991.

N. Ogbonna, J. Petersen, and D. G. Dixon. Heapsim - unravelling the mathematics of heap bioleaching. In Computational Analysis in Hydrometallurgy, 35th Annual Hydrometallurgy Meeting. Canadian MetSoc, Montreal, pages 225-240, 2005.

N. Otsu. A threshold selection method from gray-level histograms. IEEE Transactions on Systems, Man and Cybernetics, 11(285-296):23-27, 1975.

J. Petersen and D. G. Dixon. Modelling zinc heap bioleaching. Hydrometallurgy, 85(2):127-143, 2007.

R. J. Roman, B. R. Benner, and G. W. Becker. Diffusion model for heap leaching and its application to scale-up. Transactions of the Society of Mining Engineers, AIME, 256(3):247-252, 1974.

K. P. Saripalli, R. J. Serne, P. D. Meyer, and B. P. McGrail. Prediction of diffusion coefficients in porous media using tortuosity factors based on interfacial areas. Groundwater, 40(4):346-352, 2002.

A. Velardo, M. Giona, A. Adrover, F. Pagnanelli, and L. Toro. Two-layer shrinking-core model: parameter estimation for the reaction order in leaching processes. Chemical Engineering Journal, 90(3):231-240, 2002. 
C. Y. Wen. Noncatalytic heterogeneous solid-fluid reaction models. Industrial E Engineering Chemistry, 60(9): $34-54,1968$.

S. Yagi and D. Kunii. Studies on combustion of carbon particles in flames and fluidized beds. In Fifth Symposium (International) on Combustion, pages 231-244. Reinhold, New York, 1955. 